\title{
Methimazole-induced hypothyroidism in rats ameliorates oxidative injury in experimental colitis
}

\author{
Ç A Íşman, B Ç Yeğen and í Alican \\ Department of Physiology, Marmara University, School of Medicine, 34716 Haydarpaşa, Ístanbul, Turkey \\ (Requests for offprints should be addressed to Í Alican, Marmara University School of Medicine, Department of Physiology, 34716 Haydarpaşa, Ístanbul, \\ Turkey; Email: incialican@yahoo.com)
}

\begin{abstract}
Depression of metabolism by hypothyroidism decreases oxidant production and thus protects tissues against oxidant damage. Moreover, it is well-known that abnormal gut motility is a common manifestation in hypo/ hyperthyroidism. In this study, we aimed to investigate the putative beneficial effects of methimazole on oxidative injury and dysmotility in a rat colitis model. Methimazole $(0 \cdot 04 \%)$ was administered in drinking water starting 15 days prior to induction of colitis. Colitis was induced by intracolonic administration of trinitrobenzene sulfonic acid $(30 \mathrm{mg} / \mathrm{ml} ; 0.8 \mathrm{ml})$ in ethanol. Six days after the induction of colitis, the fecal output was measured and used as an index for colonic motility. All rats were decapitated on the seventh day. The distal colon was weighed and the mucosal lesions were scored. Colonic lipid peroxidation (LP) and glutathione (GSH) measurements were per-
\end{abstract}

formed. The macroscopic score, the colonic wet weight and LP values of the euthyroid colitis group were found to be higher than those of the control group $(P<0 \cdot 05-0 \cdot 001)$. All these parameters were reduced in the methimazoletreated colitis group $(P<0 \cdot 01-0 \cdot 001)$. The decrease in colonic GSH levels in the colitis group was completely abolished in the methimazole-treated colitis rats $(P<0 \cdot 01)$. Induction of colitis increased the average fecal output compared with the control group $(P<0 \cdot 05)$ and methimazole in the colitis group exaggerated the fecal output $(P<0 \cdot 001)$. In conclusion, methimazole reduces colonic oxidative injury probably due to hypometabolism, which is associated with a decrease in the production of reactive oxygen intermediates and an increase in the response of antioxidant systems.

Journal of Endocrinology (2003) 177, 471-476

\section{Introduction}

There is a good deal of evidence to suggest that thyroid state may alter the induction and course of various inflammatory responses in the body. One of the major effects of thyroid hormone is to increase mitochondrial respiration through changes in the number and activity of mitochondrial respiratory chain components (Roodyn et al. 1965, Nishiki et al. 1978). A hypermetabolic state due to hyperthyroidism has been shown to accelerate reactive oxygen metabolite production in the mitochondria and to induce changes in the antioxidant system of the tissues (Asayama \& Kato 1990, Venditti et al. 1997). Hypermetabolism induced by thyrotoxicosis is known to aggravate oxidant-mediated tissue injury. Otherwise, metabolic depression brought about by hypothyroidism has been associated with a decrease in oxidant production and protects tissues against lipid peroxidation (Swaroo \& Ramasarma 1985, Paller 1986). Therefore, it has been suggested that the hyperthyroid state predisposes tissues to oxidant-mediated injury, whereas hypothyroidism is thought to protect tissues against oxidant damage.
The inflammatory bowel diseases (IBD), namely ulcerative colitis and Crohn's disease, are two chronic idiopathic diseases characterized by prominent intestinal inflammation. Among several experimental models of IBD, administration of an enema containing the contact sensitizing allergen trinitrobenzene sulfonic acid (TNBS) in ethanol produces an acute inflammation, which progresses to a chronic stage, that is morphologically similar to Crohn's disease (Elson et al. 1995). Despite the extensive literature related to the role of oxidant/antioxidant status in the pathogenesis of inflammatory bowel disease, little is known about the endocrine control of mucosal integrity in these inflammatory conditions and associated gastrointestinal dysfunction. Since decreased thyroid hormone production is associated with a variety of immunological manifestations and antithyroid drugs were found to be useful in the treatment of several automimmune diseases (Singer et al. 1994), the potential beneficial effects of the antithyroid drugs were tested to manipulate colitis (Oren et al. 1997). Although methimazole and propylthiouracil were shown to reduce mucosal damage in a rat model of colitis, their mode of action was not demonstrated. 
The most common gastrointestinal symptoms in hyperthyroid patients include diarrhea, malabsorption and steatorrhea while those in hypothyroid patients are constipation and gas retention (Sharma et al. 1995). Shafer et al. (1984) have suggested that abnormal gut motility is the primary cause of gastrointestinal manifestations in hypo/ hyperthyroidism. On the other hand, colitis is associated with alterations in motor function, which varies with disease activity (Goldhill et al. 1999). As inflammation progresses, there are changes in the profile of inflammatory/immune cells and associated mediators that may directly or indirectly affect smooth muscle contractility. However, the effect of antithyroid drugs on colitisinduced gastrointestinal dysmotility has not previously been tested.

Thus, the aim of this study was to evaluate the putative beneficial effects of an antithyroidal drug, methimazole, on oxidative colonic injury and dysmotility in an experimental rat colitis model.

\section{Materials and Methods}

\section{Animals}

Wistar albino rats of both sexes (220-280 g) were kept in a room at a constant temperature of $22 \pm 1{ }^{\circ} \mathrm{C}$ with a $12-\mathrm{h}$ light/12-h darkness cycle and fed standard pellet chow and water which were available ad libitum. This study was approved by Marmara University School of Medicine, Animal Care and Use Committee.

Methimazole $(0 \cdot 04 \%)$ was administered in drinking water starting 15 days prior to induction of colitis, while control animals were treated with normal tap water for the same period of time (control group). Each group consisted of 7 animals.

\section{Induction of colitis and determination of colonic damage and fecal output}

Two weeks after the induction of hypothyroidism, inflammation was induced in the colon, under light ether anesthesia, by a single intracolonic administration of $0.8 \mathrm{ml}$ of a $30 \mathrm{mg} / \mathrm{ml}$ trinitrobenzene sulfonic acid solution dissolved in 38\% ethanol in saline introduced via an $8-\mathrm{cm}$ cannula (Morris et al. 1989). Comparisons were carried out with rats administered an equal volume of saline solution intracolonically (control group). On the 6th day following the induction of colitis, stool volume (mg/day) and number of fecal pellets (number/day) were measured for a 24-h period. For each animal, average stool weight (mg/ number) was calculated and expressed as 'fecal output'. Seven days after the induction of colitis, the rats were decapitated.

Trunk blood was collected and the serum samples were stored for the determination of tri-iodothyronine $\left(\mathrm{T}_{3}\right)$ and
Table 1 Criteria for macroscopic scoring of the colonic damage

\section{Macroscopic appearance}

$\begin{array}{ll}\text { Score } & \\ 0 & \text { No damage } \\ 1 & \text { Localized hyperemia, no ulcers } \\ 2 & \text { Ulceration without hyperemia or bowel wall thickening } \\ 3 & \text { Ulceration with inflammation at one site } \\ 4 & \text { Two or more sites of ulceration/inflammation } \\ 5 & \begin{array}{l}\text { Major sites of damage extending more than } 1 \mathrm{~cm} \text { along } \\ \text { the length of colon }\end{array} \\ 6-10 & \begin{array}{l}\text { If damage extends more than } 2 \mathrm{~cm} \text { along the length } \\ \text { of colon, the score is increased by one for each } \\ \text { additional } 1 \mathrm{~cm}\end{array}\end{array}$

thyroxine $\left(\mathrm{T}_{4}\right)$ levels using radioimmunoassay. The last $8 \mathrm{~cm}$ of the colon was excised, opened longitudinally, and rinsed with saline solution. The distal colon was weighed and the mucosal lesions were examined macroscopically by a blinded observer using the criteria outlined in Table 1 (Wallace et al. 1989). Then, the colonic samples were stored at $-80^{\circ} \mathrm{C}$ for the measurement of lipid peroxidation and glutathione levels.

\section{Determination of lipid peroxidation and glutathione levels}

Tissue samples were homogenized in $10 \mathrm{ml}$ vol. of icecold $10 \%$ trichloracetic acid, in an Ultra Turrax tissue homogenizer. Homogenized tissue samples were centrifuged at 3000 r.p.m. for $15 \mathrm{~min}$ at $4{ }^{\circ} \mathrm{C}$. The supernatant was removed and recentrifuged at 15000 r.p.m. for $8 \mathrm{~min}$. Glutathione measurements were performed using a modification of the Ellman procedure (Aykaç et al. 1985). Lipid peroxidation was quantified by measuring the formation of thiobarbituric acid-reactive substances as described previously. Lipid peroxide levels were expressed in nmol malondialdehyde (MDA) per g tissue (Casini et al. 1986).

\section{Statistical analysis}

Data were expressed as means \pm S.E.M. Groups of data were analyzed by one-way analysis of variance followed by Tukey-Kramer multiple-comparisons test. Calculations were performed using an Instat statistical analysis package (GraphPad Software, San Diego, CA, USA). P values $<0.05$ were regarded as significant.

\section{Results}

Methimazole-treated rats were hypothyroid, as confirmed by serum $\mathrm{T}_{3}$ and $\mathrm{T}_{4}$ values $(25.5 \pm 0.29 \mathrm{ng} / \mathrm{dl}$ and $0 \cdot 49 \pm 0.01 \mathrm{ng} / \mathrm{dl}$ respectively) which were significantly 


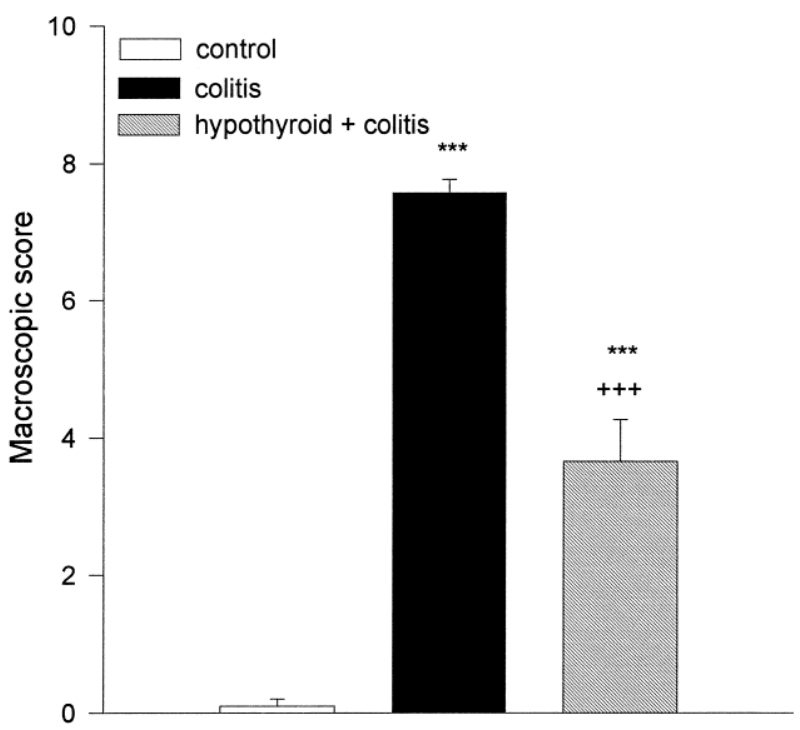

Figure 1 The macroscopic damage score of control, colitis and hypothyroid colitis groups. Values are means \pm S.E.M. of 7 rats. ${ }^{* *} P<0 \cdot 001$, compared with the control group; ${ }^{++} P<0 \cdot 001$, compared with the colitis group.

lower than those of control rats $(54.75 \pm 0.75 \mathrm{ng} / \mathrm{dl}$ and $6 \cdot 48 \pm 0 \cdot 46 \mathrm{ng} / \mathrm{dl}$ respectively; $P<0 \cdot 001)$.

\section{Macroscopic damage score}

The macroscopic damage score in the euthyroid colitis group $(7 \cdot 57 \pm 0 \cdot 2)$ was found to be significantly higher than that of the control group $(0 \cdot 1 \pm 0 \cdot 1 ; \quad P<0 \cdot 001)$, while the colitis score was reduced when the rats were hypothyroid (3.66 $\pm 0 \cdot 6 ; P<0 \cdot 001)$ (Fig. 1).

\section{Colonic wet weight}

The colonic wet weight ( $\mathrm{g} / 100 \mathrm{~g}$ body weight) of the euthyroid-colitis group $(0.99 \pm 0.07 \mathrm{~g})$ was significantly higher than that of the control group $(0 \cdot 21 \pm 0 \cdot 01 \mathrm{~g}$; $P<0 \cdot 001)$. Methimazole pretreatment before the induction of colitis markedly diminished the wet weight of colonic tissue $(0 \cdot 61 \pm 0.06 \mathrm{~g} ; P<0 \cdot 01)$.

\section{Lipid peroxidation and glutathione levels}

Colonic lipid peroxidation level in the colitis group $(42 \cdot 9 \pm 1 \cdot 01 \mathrm{nmol} \mathrm{MDA} / \mathrm{g})$ was significantly higher than that in the control group $(32 \cdot 2 \pm 1.10 \mathrm{nmol} \mathrm{MDA} / \mathrm{g}$; $P<0 \cdot 05)$. In hypothyroid rats with colitis, the lipid peroxidation level $(30 \cdot 43 \pm 4 \cdot 10 \mathrm{nmol} \mathrm{MDA} / \mathrm{g})$ was not significantly different from the control group (Fig. 2). The colonic glutathione level was decreased in the colitis group $(0.64 \pm 0.09 \mu \mathrm{mol} / \mathrm{g})$ as compared with controls $(2.94 \pm 0.16 \mu \mathrm{mol} / \mathrm{g} ; P<0 \cdot 001)$, while this reduction was

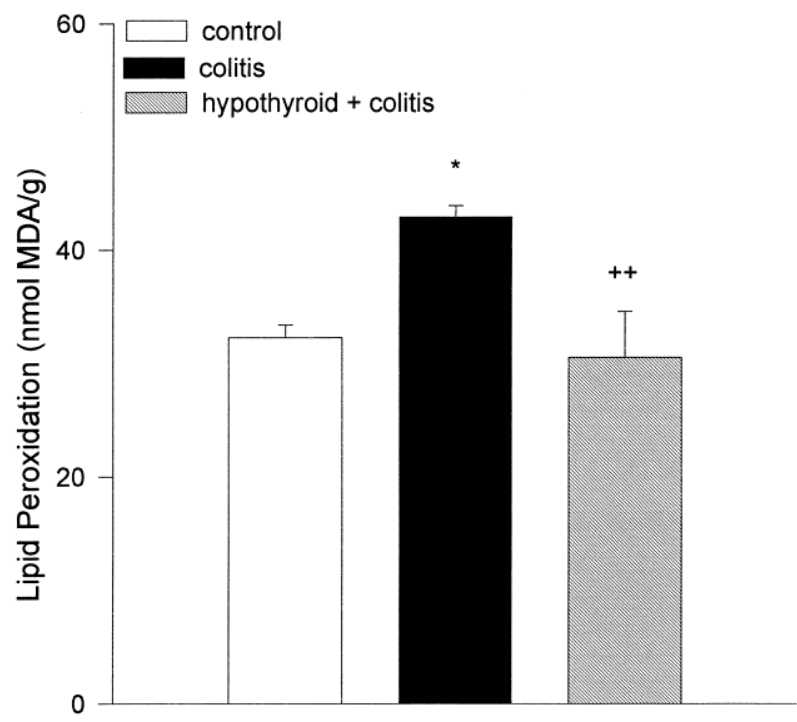

Figure 2 The colonic lipid peroxidation levels of control, colitis and hypothyroid colitis groups. Values are means \pm S.E.M. of 7 rats. ${ }^{*} P<0 \cdot 05$, compared with the control group; ${ }^{++} P<0 \cdot 01$, compared with the colitis group.

completely abolished in hypothyroid rats with colitis $(2 \cdot 49 \pm 0 \cdot 14 \mu \mathrm{mol} / \mathrm{g} ; P<0 \cdot 001)$ (Fig. 3).

\section{Fecal output}

Induction of colitis increased the daily stool volume $(12.0 \pm 0.6 \mathrm{~g} /$ day; $P<0 \cdot 01)$, without any effect on the daily fecal pellet number $(40 \cdot 1 \pm 1 \cdot 6 /$ day $)$, when

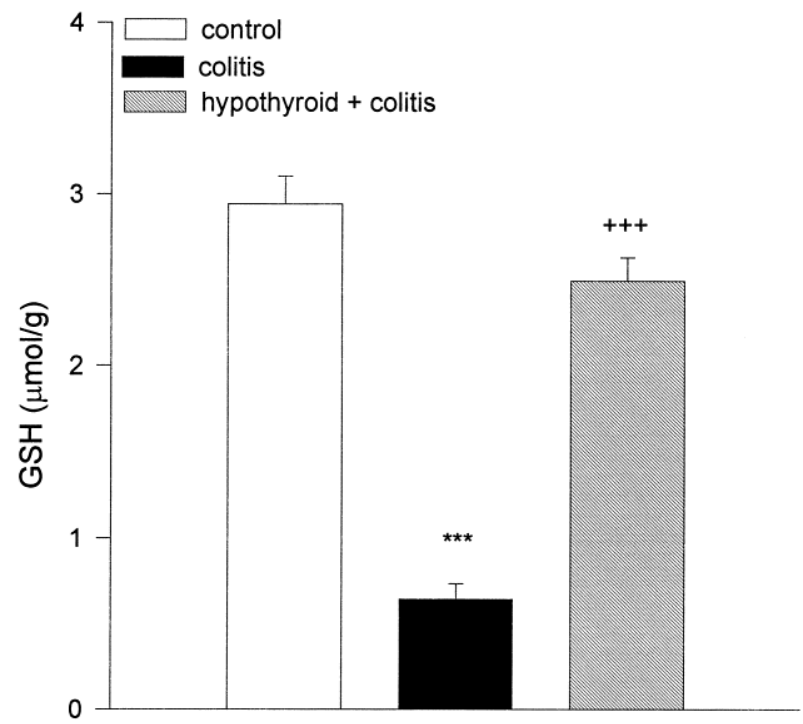

Figure 3 The colonic glutathione (GSH) levels of control, colitis and hypothyroid colitis groups. Values are means \pm S.E.M. of 7 rats. ${ }^{* * *} P<0 \cdot 001$, compared with the control group; ${ }^{+++} P<0 \cdot 001$, compared with the colitis group. 


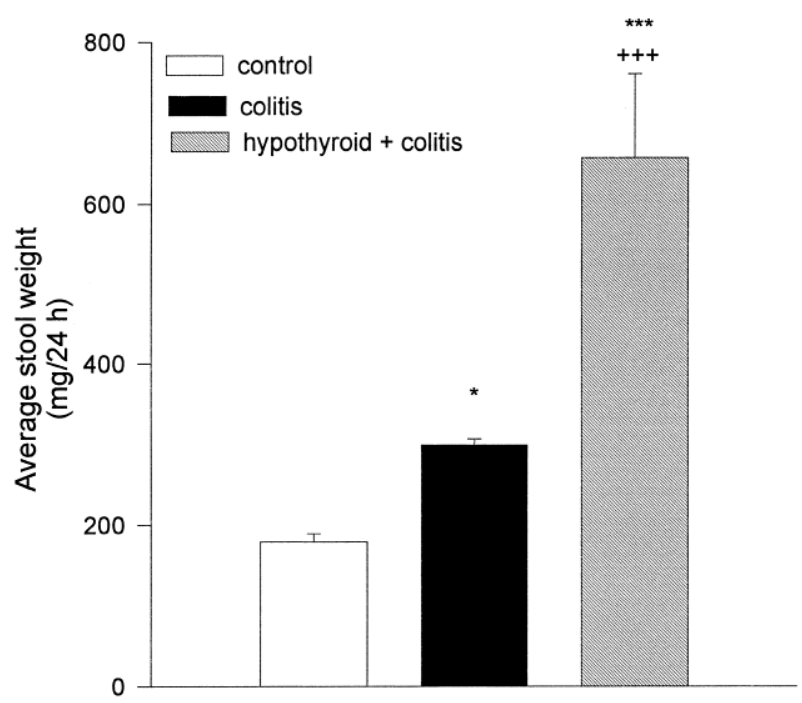

Figure 4 Average stool weight of control, colitis and hypothyroid+colitis groups. Values are means \pm S.E.M. of 7 rats. ${ }^{*} P<0.05$ and ${ }^{* * *} P<0 \cdot 001$, compared with the control group; ${ }^{+++} P<0 \cdot 001$, compared with the colitis group.

compared with the control group $(6 \cdot 6 \pm 0 \cdot 5 \mathrm{~g} /$ day and $38 \cdot 3 \pm 3 \cdot 1 \mathrm{~g} /$ day respectively). Since the volume of fecal pellets was reduced in colitis without a change in number, a significant increase in average fecal output $(300 \cdot 0 \pm 7 \cdot 2 \mathrm{mg} /$ pellet) was observed as compared with the control group $(179 \cdot 0 \pm 10 \cdot 1 \mathrm{mg} /$ pellet; $P<0 \cdot 05)$ (Fig. 4). While administration of methimazole for two weeks had no significant effect on daily fecal output $(281 \cdot 0 \pm 22 \cdot 2 \mathrm{mg} /$ pellet, not shown on the Figure) with respect to the control group, a reduction in the number of fecal pellets was observed $(26 \cdot 8 \pm 1 \cdot 5 /$ day; $P<0 \cdot 01)$. However, methimazole in the colitis group exaggerated the fecal output $(657 \cdot 7 \pm 103.7 \mathrm{mg} /$ pellet; $P<0 \cdot 001)$ as shown by the increased daily stool volume $(13 \cdot 2 \pm 1.7 \mathrm{~g} /$ day), despite the reduction in the number of fecal pellets $(21 \cdot 0 \pm 1 \cdot 7 /$ day $)$.

\section{Discussion}

The present study demonstrates that hypothyroidism induced by methimazole significantly ameliorates the inflammatory responses in TNBS-induced experimental colitis in the rat. This was shown by significant decreases in the macroscopic damage score and tissue wet weight in the hypothyroid group as compared with euthyroids. These data are in accordance with previous findings showing that the colonic damage was decreased by antithyroid drugs as shown by lesion score, tissue weight and myeloperoxidase staining both in early and late phases (Oren et al. 1997). Additionally, we observed that increased lipid peroxidation and glutathione utilization in the colitis group were reversed by methimazole treatment. These latter findings suggest that the colonic oxidant/ antioxidant status has been altered by hypothyroidism.

Methimazole has frequently been used in the treatment of Graves' disease and has been efficacious in experimental systemic lupus erythematosus in mice, both of which are autoimmune disorders (Singer et al. 1994). Several lines of evidence suggest that this mechanism of action may be attributed to the effect of hypothyroidism on cell-mediated immunity. For example, decreased thyroid function has been shown to be associated with CD4 T cell activation, increased number and activation of CD8 cells and decreased interleukin-2 (Volper 1994). Methimazoleinduced hypothyroidism has been shown to suppress the expresssion of tumor necrosis factor (TNF) gene in peritoneal macrophages and to reduce alveolar macrophage production upon stimulation with lipopolysaccharide (Liu \& $\mathrm{Ng}$ 1991, Liu 1993, Liu et al. 1993). However, in thioacetamide-induced hepatic failure, the suppression of TNF- $\alpha$ and other proinflammatory cytokines has not played a key role in the prevention of liver damage by hypothyroidism (Bruck et al. 1998). Thus, it was suggested that hypometabolism and decreased hepatocyte oxygen demand, associated with hypothyroidism, might be protective for the insulted liver (Bruck et al. 1998). Since methimazole ameliorates the TNBS-induced colonic damage, the results of the present study, taken together with the aforementioned studies, suggest that hypothyroidism might slow the colonic inflammatory processes by reducing the tissue oxygen demand, and some decrease in thyroid function appears to be beneficial for the inflamed colonic tissue.

One of the major effects of thyroid hormone is to increase mitochondrial respiration (Nishiki et al. 1978) which results in increased generation of reactive oxygen species, leading to oxidative damage to membrane lipids. There is a good deal of evidence to indicate that metabolic depression brought about by hypothyroidism is associated with a decrease in free radical production and a subsequent protection against lipid peroxidation (Asayama et al. 1987). This supports the notion that reduced demand for oxygen in hypothyroidism serves as a protective factor in tissue injury due to reactive oxygen metabolites. In agreement with these data, our results demonstrate that colitis induces oxidative damage associated with a reduction in glutathione reserves, whereas hypothyroidism offers a protection against free radical damage with a greater response of the antioxidant systems. This in vivo finding raised the possibility that methimazole could have a direct antioxidant effect. In an attempt to clarify this possibility, we compared the luminol-enhanced chemiluminescence values of xanthine-xanthine oxidase in the absence and presence of methimazole $\left(10^{-9}-10^{-3} \mathrm{M}\right)$ and our results revealed that methimazole had no superoxide scavenging activity per se (authors' unpublished observation). Therefore, the protective mechanism may be related to the effects of 
hypometabolism on the production of reactive oxygen intermediates.

Hypothyroid patients frequently complain of moderate or severe constipation and this hypomotility may well be an early physical manifestation of hypothyroidism (Rahman et al. 1991). On the other hand, prominent clinical features of IBD are diarrhea and abdominal cramping (Rao \& Read 1990). It has been reported that thyrotoxicosis may exacerbate the diarrhea of colitis and/or may cause patients to be refractory to treatment (Jarnerot et al. 1975). Thus, it is likely that the antithyroid drugs have an inhibitory effect on colitis-induced hypermotility. In the present study, colitis led to increased daily fecal volume without a change in the frequency of defecation, which resulted in an increased average stool weight (volume/pellet). On the other hand, when methimazole was given to the colitis group, frequency of fecal discharge was reduced, while colitis-induced increase in daily stool volume was not altered; thus, the average stool weight in $24 \mathrm{~h}$ was found to be increased twofold. This finding suggests that antithyroid treatment may be beneficial in the alleviation of colitis-induced diarrhea, without an effect on total stool volume. Since methimazole was effective in improving the oxidant colonic injury, it appears that the changes in the profile of inflammatory/ immune cells and associated mediators have affected the colonic contractile activity. Moreover, hypothyroidism may influence the function of the muscle cell indirectly, by affecting the composition of connective tissue and extracellular space, and/or directly, by altering metabolic pumps and movement of electrolytes (Kowalewski \& Kolodej 1977).

Therefore, the present study demonstrates that methimazole reduces colonic oxidative injury. This protective mechanism may be due to hypometabolism, which is associated with a decrease in the production of reactive oxygen intermediates and an increase in the response of antioxidant defence systems. In conclusion, the concomitant presence of hypothyroidism or the use of antithyroid drugs in IBD might be protective against inflammatory challenges of the colon, while the diarrhea of colitis might be exacerbated.

\section{Acknowledgement}

The authors thank Eczacibasi Scientific Research and Award Fund for their support of this work.

\section{References}

Asayama K \& Kato K 1990 Oxidative muscular injury and its relevance to hyperthyroidism. Free Radical Biology and Medicine $\mathbf{8}$ 293-303.

Asayama K, Dobashi K, Hayashibe H, Megata Y \& Kat K 1987 Lipid peroxidation and free radical scavengers in thyroid dysfunction in the rat: a possible mechanism of injury to heart and skeletal muscle in hyperthyroidism. Endocrinology 1231 2112-2118.
Aykaç G, Uysal M, Yalçin AS, Koçak-Toker N, Sivas A \& Öz H 1985 The effect of chronic ethanol ingestion on hepatic lipid peroxide, glutathione, glutathione peroxidase and glutathione transferase in rats. Toxicology 36 71-76.

Bruck R, Oren R, Shirin H, Aeed H, Papa M, Matus Z, Zaidel L, Avni Y \& Halpern Z 1998 Hypothyroidism minimizes liver damage and improves survival in rats with thioacetamide-induced fulminant hepatic failure. Hepatology 27 1013-1020.

Casini A, Ferrali M, Pompella AS, Maellaro E \& Comporti M 1986 Lipid peroxidation and cellular damage in extrahepatic tissues of bromobenzene toxicated mice. American Journal of Pathology 123 $520-531$.

Elson CO, Sartor RB, Tennyson GS \& Riddell RH 1995 Experimental models of inflammatory bowel disease. Gastroenterology 109 1344-1367.

Goldhill JM, Bossone C, Pineiro-Carrero V \& Shea-Donohue T 1999 Progressive alterations in circular smooth muscle contractility in TNBS-induced colitis in rats. Neurogastroenterology and Motility 11 347-356.

Jarnerot G, Azas Khan AK \& Truelove S 1975 The thyroid in ulcerative colitis and Crohn's disease. II. Thyroid enlargement and hyperthyroidism in ulcerative colitis. Acta Medica Scandinavica 197 83-87.

Kowalewski K \& Kolodej A 1977 Myoelectrical and mechanical activity of stomach and intestine in hypothyroid dogs. Digestive Diseases and Sciences 22 235-240.

Liu WK 1993 Expression of tumor necrosis factor and c fos genes in peritoneal macrophages of hypothyroid mice. Inflammation $\mathbf{1 7}$ 217-225.

Liu WK \& Ng TB 1991 Effect of methimazole-induced hypothyroidism on alveolar macrophages. Virchows Archiv B, Cell Pathology including Molecular Pathology 60 21-26.

Liu WK, Tsui KW \& Wong CC 1993 Repressed activity of peritoneal macrophages of hypothyroid mice. Virchows Archiv B, Cell Pathology including Molecular Pathology 63 131-136.

Morris GP, Beck PL, Herridge MS, Depew WT, Szewczuk MR \& Wallace JL 1989 A hapten-induced model chronic inflammation and ulceration in the rat colon. Gastroenterology 96 795-803.

Nishiki K, Ericinska M, Wilson DF \& Cooper S 1978 Evaluation of oxidative phosphorylation in hearts from euthyroid, hypothyroid and hyperthyroid rats. American Journal of Physiology 235 C212-C219.

Oren R, Maaravi Y, Karmeli F, Kenet G, Zeidel L, Hubert A \& Eliakim R 1997 Anti-thyroid drugs decrease mucosal damage in a rat model of experimental colitis. Alimentary Pharmacology and Therapeutics 11 341-345.

Paller MS 1986 Hypothyroidism protects against free radical damage in ischemic acute renal failure. Kidney International 29 1162-1166.

Rahman Q, Haboubi NY, Hudson PR, Lee GS \& Shah IU 1991 The effect of thyroxine on small intestinal motility in the elderly. Clinical Endocrinology 35 443-446.

Rao SS \& Read NW 1990 Gastrointestinal motility in patients with ulcerative colitis. Scandinavian Journal of Gastroenterology 172 (Suppl) $22-28$

Roodyn DB, Freeman KB \& Tata JR 1965 The stimulation by treatment in vivo with tri-iodothyronine or amino acid incorporation into protein by isolated rat liver mitochondria. Biochemical Journal 94 628-641.

Shafer RB, Prentiss RA \& Bond JH 1984 Gastointestinal transit in thyroid disease. Gastroenterology 86 852-855.

Sharma S, Longo WE, Baniadam B \& Vernava AM 1995 Colorectal manifestations of endocrine disease. Diseases of the Colon and Rectum 38 318-323

Singer DS, Kohn LD, Zinger H \& Mozs E 1994 Methimazole prevents induction of experimental systemic lupus erythematosus in mice. Journal of Immunology 153 873-880. 
Swaroo A \& Ramasarma T 1985 Heat exposure and hypothyroid conditions decrease hydrogen peroxide generation in liver mitochondria. Biochemical Journal 226 403-408.

Venditti P, Balsetrieri A, Di Meo S \& De Leo T 1997 Effect of thyroid state on lipid peroxidation, antioxidant defences, and susceptibility to oxidative stress in rat tissues. Journal of Endocrinology 155 151-157.

Volper R 1994 Evidence that the immunosuppressive effects of antithyroid drugs are mediated through actions on the thyroid cell, modulating thyrocyte-immunocyte signalling: a review. Thyroid 4 145-146.
Wallace JL, Braquet P, Ibbotson GC, MacNoughton WK \& Cirino G 1989 Assessment of the role of platelet activating factor in an animal model of inflammatory bowel disease. Journal of Lipid Mediators 1 13-23.

Received 3 February 2003

Accepted 18 February 2003 